\title{
OBLIQUE SURFACE WAVES ON A PAIR OF PLANAR PERIODIC SLOTTED WAVEGUIDES.
}

\author{
C. Tannous \\ TRLabs, Suite 108, 15 Innovation Boulevard Saskatoon SK, S7N 2X8, Canada \\ R. Lahlou and M. Amram \\ Dpartement de Gnie physique, Ecole Polytechnique de Montral C.P. 6079, \\ Succursale A, Montral, PQ, H3C 3A7, Canada
}

(Dated: March 16, 2001)

\begin{abstract}
The dispersion relation and mode amplitudes of oblique surface waves propagating on an acoustic double comb filter are obtained with a method based on the calculus of residues. We obtain a better agreement (below $480 \mathrm{~Hz}$ ) between theoretical predictions and measurements reported previously when the filter was being supposed to be made of a single comb structure.
\end{abstract}

*Electronic address: tannous@univ-brest.fr; Present address: Laboratoire de Magntisme de Bretagne, UPRES A CNRS 6135, Universit de Bretagne Occidentale, BP: 809 Brest CEDEX, 29285 FRANCE 


\section{INTRODUCTION}

The behavior of a slow wave filter made of a pair of planar periodic waveguides subjected to low frequency acoustic waves incident upon the aperture separating the waveguides has been investigated theoretically and experimentally for its potential use in acoustic filtering devices [1]. Each waveguide has a comb structure consisting of a periodic array of blades perpendicular to a base plane (Figure 1).

Using a mathematical model borrowed from the study of electrical filters, a filter having the same geometric structure of a single comb waveguide has been analyzed previously [1]. The dispersion relation, amplitude and phase as functions of frequency and wave number were derived and compared to experiment. In this work, we extend our previous theoretical results and consider the actual nature of the filter consisting of the two waveguides facing each other. We derive the dispersion relation and reflection (transmission) coefficients of surface waves propagating along any oblique wave number in the plane parallel to the comb structure base planes.

Our calculations are based on a weak-coupling approximation and in the limit of large distance separating the two structures. This means the separation is much larger than the inter-blade distance. The blades are supposed to have a vanishingly small thickness and we neglect possible reflections from the planar base affecting the propagating modes, by direct analogy with the electromagnetic case [2]. This is equivalent to assuming a slot depth large with respect to the inverse lowest attenuation of the structure [2].

Our work is organized as follows: In section II, we discuss the geometry, propagating modes dispersion and amplitude relation for the surface waves. Section III covers the comparison with the experimental results and the conclusion is in Section IV.

\section{DISPERSION RELATION, MODES AND AMPLITUDES}

Periodic arrays of slotted waveguides stacked to form a rectangular [3] or prismatic [4] structure are good candidates for reducing environmental noise (0.1 to $2 \mathrm{kHz})$. Their 
properties have been analyzed theoretically and experimentally $[1,3,4]$ such as their reflection scattering of sound waves harmful to the general population living near highways or other sources of damaging sources of low frequency noise. It is important to understand how these structures absorb, reflect, transmit or phase delay the incoming sound waves reaching them with arbitrary time dependent angles. For the rectangular structure, we have already undertaken such study from the experimental point of view as well as from the theoretical one. In this work, we set out to investigate a new type of structure introduced in detail in Ref. 1 theoretically and experimentally (Fig. 1).

We have studied the dispersion relation of acoustic waves impinging on the structure at an arbitrary fixed angle in the base plane, and measured the sound reflection and transmission with respect to the incident angle. Our prior theoretical investigation took account of a single comb structure only. Here we extend it and deal with a symmetrical weakly- coupled double comb structure [5] in the limit $\frac{b}{d} \gg 1$ where $\mathrm{b}$ is half the distance between the tip of the blades belonging to each of the waveguides and $\mathrm{d}$ is the inter-blade distance in any waveguide (Fig. 1).

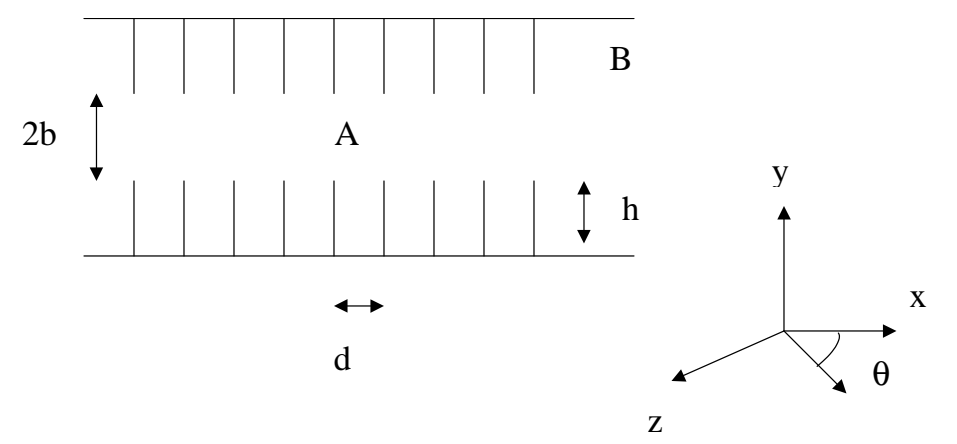

FIG. 1: Geometry of the double comb structure waveguide.

Following our notation [1], we write for the acoustic fields in region A (free space) keeping the symmetric modes only:

$$
\Phi_{A}(x, y, z)=\sum_{n=-\infty}^{\infty} A_{n} e^{-j \beta_{n} x-j \tau z} \cosh \left(\alpha_{n} y\right)
$$

where $\beta_{n}$ and $\tau$ are the propagation constants along $\mathrm{x}$ and $\mathrm{z}$ and $\alpha_{n}$ is the attenuation constant along y. The propagation constant $\beta_{0}$ defining the fundamental mode is determined 
from the propagation geometry (Fig. 1 of [6]). It is equal to $\frac{\tau}{\tan (\theta)}$ where $\theta$ is the angle, the surface wave vector makes with the $\mathrm{x}$-axis [Fig. 1]. The surface wave has a smaller velocity than in true free space by the ratio $\sqrt{\beta^{2}+\tau^{2}} / k$. In region $\mathrm{B}$, the acoustic field in the $\mathrm{n}$-th slot defined by the inequalities: $\nu d-\frac{d}{2} \leq x \leq \nu d+\frac{d}{2}$ is given by:

$$
\Phi_{B}^{\nu}(x, y, z)=\sum_{n=-\infty}^{\infty} B_{m}^{\nu} e^{-j \tau z} \cos \left(\frac{m \pi x_{\nu}}{d}\right) \cos \left[\gamma_{m}(y+b+h)\right]
$$

The coefficients $B_{m}^{\nu}$ are determined with the help of Floquet's [7] theorem $B_{m}^{\nu}=$ $B_{m} e^{-j \nu \beta_{0} d}$ and the abscissae $x_{\nu}$ are equal to $\mathrm{x}-(\nu-1 / 2) \mathrm{d}$. In order to find the dispersion equation of the surface waves and the coefficients $A_{m}, B_{m}$, we will proceed as we did in our previous work following the approach pioneered by Whitehead [7] and Hurd [2]. It consists of writing the equations of continuity for the fields $\Phi_{A}$ and $\Phi_{B}$ and their derivatives along the vertical y axis on the boundaries $y= \pm b$. These equations are considered as originating from Cauchy's theorem of residues for a meromorphic function $f(w)$ taken along some contour and the contribution of each pole is identified with the contribution of some corresponding mode. The contour and $f(w)$ should be such that the presumed theorem of residues is satisfied. Moreover, the asymptotic behavior of $f(w)$ is tailored by the underlying physical problem and is basically dictated by the scattering of the waves by the edges of the blades [7]. We obtain the following meromorphic function $f(w)$ of the complex variable $w$ :

$$
f(w)=-\frac{d B_{0} \gamma_{0} e^{-j \gamma_{0} h}}{\left[e^{-j \beta_{0} d}-1\right]}\left(\frac{j \gamma_{0}-\alpha_{0}}{w-\alpha_{0}}\right) \frac{\prod_{1}(w)}{\prod_{1}\left(j \gamma_{0}\right)} \frac{\prod_{2}\left(j \gamma_{0}\right)}{\prod_{2}(w)} \exp \left[\left(j \gamma_{0}-w\right) \frac{d \ln (2)}{\pi}\right]
$$

where $\prod_{1}(w)$ and $\prod_{2}(w)$ are the following infinite products:

$$
\prod_{1}(w)=\prod_{p=1}^{\infty}\left(w-j \gamma_{p}\right)\left(-\frac{d}{p \pi}\right) e^{\frac{d w}{p \pi}}
$$

and:

$$
\prod_{2}(w)=\prod_{p=1}^{\infty}\left(w-\alpha_{p}\right)\left(w+\alpha_{-p}\right)\left(\frac{d}{2 p \pi}\right)^{2} e^{\frac{d w}{p \pi}}
$$

The propagation constants $\gamma_{m}$ along $\mathrm{y}$, are given by:

$$
\gamma_{m}^{2}=k^{2}-\tau^{2}-\left(\frac{m \pi}{d}\right)^{2} \quad \text { with } m=0,1 \ldots
$$

In order to derive the dispersion relation, we form the ratio: 


$$
\frac{f\left(-j \gamma_{0}\right)}{f\left(j \gamma_{0}\right)}=-e^{2 j \gamma_{0} h}
$$

Taking the logarithm and using trigonometric identities [Ref. 2], we obtain:

$$
\begin{gathered}
\gamma_{0} h-\gamma_{0} \frac{d \ln (2)}{\pi}=\frac{\pi}{2}-\sin ^{-1}\left(\frac{\gamma_{0}}{\beta_{0}}\right)+\sum_{p=1}^{\infty}\left[\tan ^{-1}\left(\frac{\gamma_{0}}{\alpha_{-p}}\right)+\frac{d \gamma_{0}}{2 \pi p}\right]+\sum_{p=1}^{\infty}\left[\tan ^{-1}\left(\frac{\gamma_{0}}{\left|\gamma_{p}\right|}\right)-\frac{d \gamma_{0}}{p \pi}\right] \\
-\sum_{p=1}^{\infty}\left[\sin ^{-1}\left(\frac{\gamma_{0}}{\beta_{p}}\right)-\frac{d \gamma_{0}}{2 \pi p}\right]
\end{gathered}
$$

This equation is the same as that obtained by Hougardy and Hansen [6] who treated a single comb structure from the electromagnetic point of view. Here, we are dealing with the weak coupling symmetric case limit and with the additional simplifying assumptions: $\frac{b}{d} \gg 1, \alpha_{0} b \gg 1$ and $\alpha_{-p} \sim \beta_{-p}$. We find that the dispersion relation is essentially the same as in the case of a single comb structure. The double comb structure simply behaves as a single one from the dispersion relation point of view. This justifies our assumptions in Ref. 1 where we found very good agreement between theory and experiment up to frequencies on the order of $400 \mathrm{~Hz}$. Nevertheless this is not true for reflection (transmission) coefficients of the single/double comb structures as discussed below.

In order to calculate the mode amplitudes and obtain from them the reflection (transmission) coefficients of the structure, we use the residue of $f(w)$ at $w=\alpha_{n}$ :

$$
\operatorname{Res}[f(w)]_{w=\alpha_{n}}=A_{n} \beta_{n} e^{j \beta_{n} d / 2} \cosh \left(\alpha_{n} b\right)
$$

to obtain $(\mathrm{n}=0,1,2 \ldots)$ :

$$
\frac{\left|A_{n}\right|}{\left|B_{0}\right|}=\frac{d \gamma_{0} e^{\alpha_{n} b}}{16 \pi \cosh \left(\alpha_{n} b\right)} \frac{\left|\alpha_{n}+\alpha_{0}\right|}{\left|\alpha_{n} \beta_{n}\right|} \frac{\left|\alpha_{n}+\alpha_{1}\right|\left|\alpha_{n}-\alpha_{-1}\right|}{\left|\alpha_{n}+j \gamma_{1}\right|} \frac{\Gamma\left[2+\frac{d \alpha_{n}}{\pi}\right] \exp \left(-\frac{\alpha_{n} d \ln (2)}{\pi}\right)}{\Gamma\left[2+\frac{d}{2 \pi}\left(\alpha_{n}+\beta_{0}\right)\right] \Gamma\left[2+\frac{d}{2 \pi}\left(\alpha_{n}-\beta_{0}\right)\right]}
$$

where $\Gamma$ stands for the Euler Gamma function. For negative values of $n$, it suffices to change $\alpha_{n}$ into $-\alpha_{n}$ in the above expression. Let us note that when the separation $2 \mathrm{~b}$ between the two parts of the structure, becomes very large we recover exactly the expression found by Hougardy and Hansen [6] corresponding to a single comb structure. 
In order to calculate the $B_{n}$ coefficients, we use:

$$
f\left(-j \gamma_{n}\right)=\frac{d}{2} \frac{B_{n} \gamma_{n} e^{j \gamma_{n} h}}{\left[(-)^{n} \exp \left(-j \beta_{0} d\right)-1\right]}
$$

and the definition $(3)$ of $f(w)$ to obtain:

$$
\frac{\left|B_{n}\right|}{\left|B_{0}\right|}=\frac{2 \gamma_{0} \epsilon}{\left|\gamma_{n} e^{j \gamma_{n} h}\right|} \frac{\left|j \gamma_{0}-\alpha_{0}\right|}{\left|j \gamma_{n}+\alpha_{0}\right|} \frac{\left|\prod_{1}\left(-j \gamma_{n}\right)\right|}{\left|\prod_{2}\left(-j \gamma_{n}\right)\right|} \frac{\sin \left(\frac{\beta_{0} d}{2}\right)}{\left(\frac{\beta_{0} d}{2}\right)} \exp \left(\frac{j \gamma_{n} d \ln (2)}{\pi}\right)
$$

where $\epsilon=1$ for $n$ even, and $\epsilon=\frac{1}{\left|\tan \left(\frac{\left.\beta_{0} d\right)}{2}\right)\right|}$ for $\mathrm{n}$ odd.

Let us note that the $B_{n}$ coefficients are the same as those obtained by Hougardy and Hansen [6] reflecting the fact, the weak- coupling approximation affects in a different way the $A_{n}$ and the $B_{n}$ coefficients. This has important implications on our measurements of the amplitude profile.

\section{COMPARISON WITH EXPERIMENT}

In our previous work, we derived the dispersion relation, transmission and reflection coefficients and found excellent agreement between the single comb structure theory and experiment up to $400 \mathrm{~Hz}$ [1]. This work shows that a weak coupling between two comb structures does not affect the surface wave dispersion relations and the $B_{n}$ amplitude coefficients but it does affect the $A_{n}$ amplitude coefficients.

We are going to evaluate how our theory modifies the amplitude ratio $\frac{\left|A_{0}\right|}{\left|B_{0}\right|}$ associated with the fundamental mode $(n=0)$ in relation (10) compared with same given in Lahlou et al. [1]. The double comb over single comb structure ratio of the two expressions is given by:

$$
F(\theta)=\frac{e^{\alpha_{0} b}}{2 \cosh \left(\alpha_{0} b\right)}
$$

For a given frequency and a given incident angle $\theta$ we solve the dispersion relation given by equation (8), obtain the propagation factor $\alpha_{0}$ and use it in (13). The corrections $\mathrm{F}(\theta)$ in $\mathrm{dB}$ are plotted versus $\theta$ in the interval $[1,80]$ degrees for various frequencies [400-600 $\mathrm{Hz}]$ in Fig.2. 


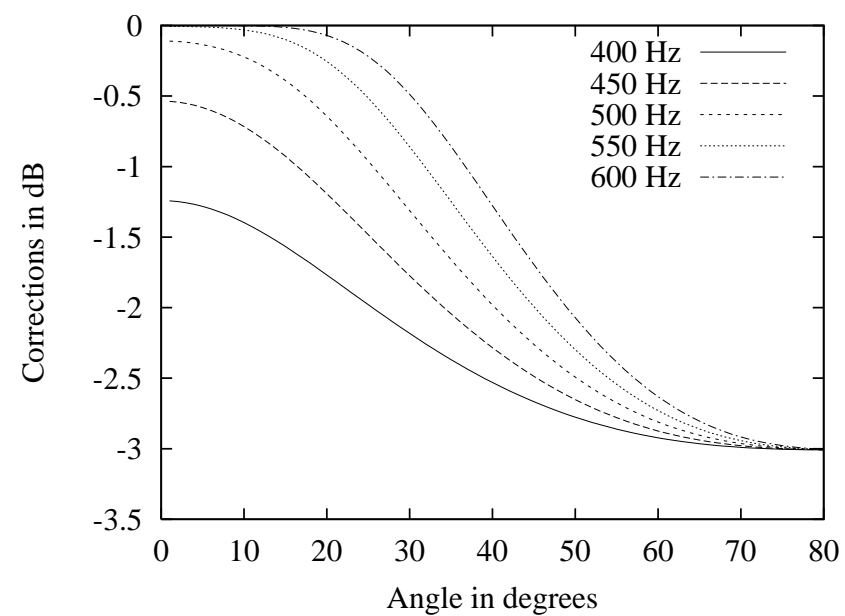

FIG. 2: Corrections $F(\theta)$ to the fundamental mode amplitude ratio $\frac{\left|A_{0}\right|}{\left|B_{0}\right|}$ with the following values (from the experimental setup) $\mathrm{b}=0.0125 \mathrm{~m}, \mathrm{~d}=0.05 \mathrm{~m}, \mathrm{~h}=0.112 \mathrm{~m}$. The corrections calculated from $10 \log _{10}\left(\frac{F(\theta=0)}{F(\theta)}\right)$ are evaluated as a function of the incident angle $\theta$ at a fixed frequency varying from 400 to $600 \mathrm{~Hz}$ by steps of $50 \mathrm{~Hz}$.

The correction comprised between 0 and $-3 \mathrm{~dB}$ is small for higher frequencies and small incident angles. It decreases rapidly for angles larger than 10 to 20 degrees and by a larger amount for higher frequencies. A comparison to the experimental data reveals that the correction is pronounced mostly at higher frequency $(336 \mathrm{~Hz})$ and for the largest angle of incidence (47 degrees). For the highest experimental frequencies (480 and $496 \mathrm{~Hz}$ ), the correction introduces more disagreement between the experimental and theoretical single comb structure theory. This behavior may be explained by the fact that there are several sources of errors associated with the measurements at these higher frequencies.

\section{CONCLUSION}

We have developed a weak coupling theory based on the calculus of residues in order to model the oblique propagation of acoustic waves propagating through a slow wave filter made of a pair of comb structured waveguides separated by a distance that is large with respect to the inter-blade distance. The correction arising from the symmetrical coupling between the two waveguides has been evaluated and shown to improve slightly the agreement between the theoretical and the experimental values of Lahlou et al. [1] being at the most $3 \mathrm{~dB}$ for 
the largest frequency and angle evaluated. Those results show that the approximation taken in our previous investigation is quite acceptable and that the new theory does not bring substantial additional accuracy to our previous single comb structure model. Our studies of the strong coupling case $(b<d)$ being mathematically much more complicated, and intended for improving the agreement between the theoretical results and the experimental ones at the higher frequencies are in progress and will be reported in the near future.

[1] R. Lahlou, M. Amram and G. Ostiguy, 1989, J. Acoust. Soc.Am. 85, 1449-1455, "Oblique acoustic wave propagation through a slotted waveguide".

[2] R.A. Hurd, 1954, Can. J. Phys. 32, 727-734, "Propagation of an electromagnetic wave along an infinite corrugated surface".

[3] L. Mongeau, M. Amram and J. Rousselet, 1985, J. Acoust. Soc.Am. 80, 665-671, "Scattering of sound waves by a periodic array of slotted waveguides".

[4] M. Amram and R. Stern, 1981, J. Acoust. Soc. Am. 70, 1463-1472. "Refractive and other acoustic effects produced by a prism-shaped network of rigid strips".

[5] L. Brillouin, 1948, J. Appl. Physics 19, 1023-1041. "Waveguides for slow waves".

[6] R.W. Hougardy and R.C. Hansen, 1958, IRE Trans. Antennas and Propag. AP-2, 370-376, "Scanning surface wave antenna - oblique surface waves over a corrugated conductor".

[7] E.A.N. Whitehead, 1951, Proc. IEEE 98, (III), 133-140, "Theory of parallel plate media for microwave lenses". 